\title{
Humanidad y universo digital: prolegómenos al problema ético de la utilidad y el perjuicio de lo digital para la vida*
}

\author{
Jorge Francisco Maldonado Serrano* \\ Dairon Rodríguez $^{* * *}$
}

Recibido: 10 de octubre de 2014 - Aprobado: 30 de enero de 2014

No resulta fácil determinar si la humanidad se beneficia o perjudica con la tecnología digital. Para aportar a este debate, es preciso revisar el funcionamiento del universo digital. ¿Qué estatuto puede dársele?, ¿se trata de una realidad reducible a explicaciones físicas?, ¿puede considerarse una realidad metafísica? Este primer componente del problema debe ser recorrido con la mayor minucia posible; sin embargo, comprender el funcionamiento no conlleva directamente comprender los usos de lo digital y las maneras en que la cotidianidad de lo humano puede verse transformada. Este segundo componente del problema abrirá paso al juicio ético. El tercer componente del problema es, en este orden de ideas, la relación entre el universo digital en sí y su usuario. Si es posible comprender mejor esta relación entre lo digital y la "humanidad digitalizada", ¿podríamos a partir de allí evaluar la utilidad y los perjuicios de lo digital para la vida? Solo un cuarto componente del problema nos permitirá diferenciar en qué sentido el perjuicio de la tecnología digital depende del uso que se le dé y en qué sentido el beneficio es intrínseco a ella misma. El objetivo de este artículo es recorrer estos cuatro componentes para proponer una lectura crítica del universo digital y su actualización en la sociedad.

Palabras clave: humanismo, universo digital, desarrollo tecnológico.

* Este artículo desarrolla una de las conclusiones de la Investigación 5237, "Devenires en política, economía y estética: el problema de la filosofía en Deleuze", aprobado por la VIE UIS, y prepara interdisciplinarmente un estudio acerca de la influencia de los códigos en el mundo de la vida y sobre los procesos de digitalización.

** Profesor Asociado Universidad Industrial de Santander (UIS), hace parte del Grupo de investigación Politeia. Dirección postal: Cra 27 calle 9, Bucaramanga, Colombia. Correo electrónico: jorgefcomaldonado@gmail.co

*** Profesor Auxiliar Universidad Industrial de Santander (UIS), hace parte del Grupo de investigación Politeia. Dirección postal: Cra 27 calle 9, Bucaramanga, Colombia. Correo electrónico: rodriguez.dairon@gmail.com. 


\title{
Humanity and digital universe: prefaces to the ethical problem of the utility and damage of the digital to the lifetime*
}

\author{
Jorge Francisco Maldonado Serrano** \\ Dairon Rodríguez
}

\section{Abstract}

It is not easy to determine whether humanity is benefited or harmed by the digital technology. To contribute to this debate it is necessary to check out the performance of the digital universe. What statute can it be addressed to it? Is it a reality reducible to physical explanations? Can it be considered a metaphysical reality? This first component of the issue must be analyzed with the greatest care. However, understanding the functioning does not mean that we can understand the uses of the digital and the ways in which the everyday work of the human can be transformed. This second component of the issue will open the way for the ethical trial. The third component of the issue is, in this order of ideas, the relationship between the universe digital itself and the user of it. If it is possible to understand better this relationship between the digital and the "digitized Mankind" Could we from there assess the utility and the damages of the digital for the lifetime? Only a fourth component of the issue will allow us differentiate in what sense the damage of the digital technology depends on its use and in what sense the benefit is intrinsic to it. The goal of this article this is to analyze these four components to propose a critical reading of the digital universe and its update in the society.

Keywords: Humanism, digital universe, technological development.

This paper develops one of the conclusions of Research 5237, "Becomings in politics, economy and aesthetics: the problem of philosophy in Deleuze" approved by the UIS VIE and preparing an interdisciplinary study on the influence of the codes in the world of life and the processes of digitalization.

* Associate Professor at the Universidad Industrial de Santander (UIS); is a member of the Research Group Politeia Address: Cra 27 calle 9, Bucaramanga, Colombia. Email: jorgefcomaldonado@gmail.com.

.*. Assistant Professor at the Universidad Industrial de Santander (UIS); is a member of the Research Group Politeia. Address: Cra 27 calle 9, Bucaramanga, Colombia. Email: rodriguez.dairon@gmail.com. 


\title{
Humanisme et l'univers digital: des prolégomènes au problème éthique de l'utilité et le préjudice du digital pour la vie*
}

\author{
Jorge Francisco Maldonado Serrano* \\ Dairon Rodrígue***
}

\section{Résumé}

Il ne semble pas facile de déterminer si l'humanité se bénéficie ou se nuit avec la technologie digitale. Pour fournir à ce débat, il est précis de réviser le fonctionnement de l'univers digital. Quel statut peut-il être donné?: L'univers digital s'agit-il d'une réalité réductible aux explications physiques? Une réalité peut-elle se considérer métaphysique? Ce premier composant du problème doit être parcouru avec la plus grande minutie possible. Cependant, comprendre le fonctionnement ne comporte pas directement à comprendre les usages du digital et les manières dans lesquelles la quotidienneté de l'humain peut se montrer transformée. Ce second composant du problème ouvrira un passage au jugement éthique. Le troisième composant du problème est dans cet ordre d'idées, la relation entre l'univers digital en soi et l'utilisateur même. Si on est possible comprendre mieux cette relation entre le digital et «la humanité numérisée»: pourrions-nous à partir de celle- là évaluer l'utilité et les préjudices du digital pour la vie? Seulement un quatrième composant du problème nous permettra de différer dans quel sens le préjudice de la technologie digitale dépend de l'usage qui lui est donné et dans quel sens le bénéfice est intrinsèque à elle-même. L'objectif de cet article est parcourir ces quatre composants pour proposer une lecture critique de l'univers digital et de son actualisation dans la société.

Mots clés: Humanisme, l'univers digital, le développement technologique.

Ce texte développe l'une des conclusions de la Recherche 5237, «Devenirs dans une politique, une économie et une esthétique: le problème de la philosophie en Deleuze», approuvé par la VIE UIS, et il prépare d'une manière interdisciplinaire une étude sur l'influence des codes dans le monde de la vie, et les processus de digitalisation

* Professeur associé UIS, fait partie du Groupe de recherche Politeia. Adresse: Cra 27 calle 9, Bucaramanga, Colombia. Email: jorgefcomaldonado@gmail.com.

*** Professeur auxiliaire UIS, Groupe de recherche Politeia. Adresse: Cra 27 calle 9, Bucaramanga, Colombia. Email: rodriguez.dairon@gmail.com. 


\section{El funcionamiento del universo digital}

Sin una adecuada precisión sobre qué se entiende por lo digital, resulta difícil comprender con justicia la situación de la humanidad y de las implicaciones éticas, morales, sociales y políticas del universo digital; en última instancia, no se podría saber qué significa lo digital para la vida. Se llega a un malentendido sobre lo digital, precisamente porque no se hacen universalizaciones, categorizaciones y conceptualizaciones que distingan y relacionen la estructura de lo digital con los efectos de dicha estructura en la cotidianidad. Para proponer que hay tal o cual efecto de lo digital sobre lo cotidiano, es imprescindible analizar los componentes de lo digital y su funcionamiento de modo que pueda evidenciarse la relación entre cotidianidad y universo digital.

La estructura de esta indagación sigue el espíritu expresado por Spinoza (2000) al inicio de la tercera parte de su Ética: "De la naturaleza, y origen de los afectos", a propósito de la necesidad de comprender el continuum de las regulaciones del universo de las afecciones humanas dentro del universo físico en el que vive el hombre. Spinoza lo expresa justo al empezar el prólogo:

...[a la] mayor parte de los que han escrito sobre los afectos y la norma de vida de los hombres, no parecen tratar sobre cosas naturales, que siguen las leyes comunes de la naturaleza, sino sobre cosas que están fuera de la naturaleza. Más aun, parecen concebir al hombre en la naturaleza como un imperio en un imperio, puesto que creen que el hombre, más que seguir el orden de la naturaleza, lo perturba, y que tiene un poder absoluto sobre sus acciones, y solo por sí mismo y no por otra cosa es determinado. La causa de la impotencia e inconstancia la atribuyen, además, no al poder común de la naturaleza, sino a no sé qué vicio de la naturaleza humana, a la que por eso mismo lloran, ridiculizan y desprecian, o, como es más frecuente, detestan; y el que ha aprendido a denostar con más elocuencia o argucia la impotencia del alma humana, es tenido por divino.

Spinoza considera que desconocer esa relación lleva a un malentendido; se resume en asumir que el hombre es un imperio dentro de otro imperio, el imperio natural. Lo importante de la denuncia spinoziana radica en que recupera la continuidad explicativa entre lo natural y lo mental, evitando una proyección innecesaria de una supuesta ruptura ontológica: la res cogitans no es de diferente naturaleza que la res extensa. Si bien hay una diferencia entre lo mental y lo físico, esta no radica en el hecho de ser reinos incomunicados, sino en el hecho de ser dos expresiones de una misma realidad, de una misma substancia. Para Spinoza, solo hay una moneda con dos caras, que si bien nunca se tocan ni se ven, no pueden separarse y mantenerse sin su cara opuesta. Algo similar ocurre en lo digital cuando pensamos en su soporte físico y lo diferenciamos de lo digital como tal. 
El consenso académico y cotidiano sobre el problema ético-moral de lo digital establece una discontinuidad con lo físico porque no incluyen la comprensión de la materialidad de lo digital dentro de la interpretación que tiene de esta, de la cual extraerán conclusiones sobre lo ético-moral. De manera general, creemos que los acercamientos a la ética y lo digital pueden ubicarse en una de las siguientes cuatro asunciones:

a. Lo digital, como toda tecnología, es malo en sí mismo; los beneficios que presta son solo temporales y/o aparentes, mientras que los daños son de fondo, porque alejan al hombre de su auténtica morada (Heidegger, 2007).

b. Lo digital no es, en sí mismo, ni malo ni bueno, sino más bien el uso que se le dé se caracterizará como bueno o malo. La tecnología, se dice, podría ser utilizada para fines que dañan al otro o a la naturaleza, o para fines que la benefician (Ihde, 1990).

c. Lo digital debe controlarse y regularse en su producción y uso para que no haya perjuicios a la humanidad (Adorno, 1967).

d. Lo digital es perfectamente deseable puesto que evidencia el avance de la racionalidad del hombre y de ella misma (Searle, 2010).

Pero creemos que antes de poder asumir cualquiera de las posiciones anteriores, con lo cual expresamos que no se trata de descalificarlas y que, por el contrario, pueden permanecer unas al lado de las otras, se debe hacer un recorrido previo por la materialidad de lo digital. Para ello, proponemos partir de una idea que se ha inspirado en Spinoza y en McLuhan: lo digital hay que entenderlo como un universo dentro de nuestro mundo. ¿Por qué? Miremos.

Nuestro planeta pertenece al universo, claro está. El universo establece unas regulaciones básicas a las cuales todos los planetas estarían sometidos (por ejemplo, el espacio-tiempo, la velocidad, las partículas fundamentales, etc.). A su vez, cada planeta, como nuestro planeta Tierra, establece unas condiciones más restrictivas que las del universo y que le son propias e internas -en el universo no aplican directamente-: la gravedad, la presión atmosférica, ciertos rangos de temperatura y demás. El marco físico del universo es más amplio que el de los planetas, podría decirse que es un marco más general y menos restrictivo. Por analogía, y en este sentido, pensamos que lo digital resulta más amplio y menos condicionante que nuestro mundo. Por ello, decimos que se trata de un universo dentro de nuestro mundo. Sin embargo, suponemos una diferencia entre planeta y mundo.

Cuando utilizamos la expresión "mundo" no nos referimos a nuestro planeta, es decir, a su materialidad, sino al orden simbólico-cultural que hace que nuestra vida tenga sentido y nos consideremos humanos. Ha sido en este planeta donde se ha construido nuestro mundo, en el cual nuestra cotidianidad consiste en la manera concreta de vivirlo y experimentarlo. En tal sentido, lo digital 
debemos verlo como una extensión o ampliación del mundo; en la medida en que forma parte de este, producimos lo digital como otras creaciones culturales. Lo interesante, en este caso, es que con lo digital se ha abierto un espacio en el que quedamos liberados de las restricciones propias del mundo, y se da como un auténtico universo simbólico y cultural porque sus restricciones simbólicas resultan muy simples. La consecuencia, como ya podemos adelantar, consiste en que se abre un espacio de libertad casi ilimitada en el que cabe nuestro mundo. Esto se debe al formalismo que constituye lo digital, como veremos a continuación con cierto detalle.

Por consiguiente, cuando hablamos de universo digital, no queremos decir que se trata de un universo físico, sino de un universo formal. En este universo formal pueden acontecer muchas maneras de hacer mundos sin que por ello haya un cambio, como tal, en la formalidad del universo digital. En este universo digital las posibilidades no tienen límites, más allá de los condicionamientos lógicos, que les son inherentes. Además, es importante entender que lo digital no cambia con ninguna realización de las posibilidades que ofrece. Esto es, las condiciones de lo digital se mantienen aunque se despliegue lo digital en direcciones opuestas. Pero, ¿cómo podemos detallar este universo digital de manera que captemos toda su simpleza y potencia?

El universo digital es, en principio, una relación regulada entre ceros y unos; se trata, pues, de tres elementos. Primero, estos ceros y unos tienen una realización física concreta como lo son los flujos eléctricos, en los que el cero es su ausencia y el uno es su presencia; todo, claro está, según unas reglas de flujo. Captemos que el cero no tiene realización física propiamente, sino solo como ausencia de flujo, mientras que el uno tiene una realización física precisa, como lo es el flujo eléctrico.

El segundo elemento son las relaciones entre los flujos, que en términos físicos, son simples lapsos de millonésimas de segundo entre ocurrencias eléctricas. Físicamente, solo el uno aparece según cierto ritmo, que puede ser delimitado por el cero que no aparece físicamente. Digitalmente, el cero tiene, empero, una función específica, y tiene tanta realidad como el uno, aunque no sea así físicamente. Esta diferencia entre la ocurrencia física y la no ocurrencia, y el uno y el cero como digitales, es muy importante para entender mejor por qué no puede confundirse lo digital con lo físico que lo realiza.

El tercer elemento es precisamente la relación que mantienen los ceros y los unos: se trata de un sistema de reglas que determinan su ocurrencia. El sistema de reglas es completamente formal, si bien su realización física podría describirse rítmicamente. Es decir, una cosa es entender el flujo eléctrico como destellos ritmados y otra cosa es entender que realmente hay unas reglas que establecen dichos flujos. De nuevo, hay una diferencia entre la ritmicidad física y el sistema de reglas que propiamente regulan la aparición, porque las reglas no buscan establecer un ritmo, sino que pretenden lograr ciertos procesos, que llegamos a entender, en niveles superiores o en niveles más altos, como procesos 
matemáticos. Las ocurrencias eléctricas se determinan por una auténtica realización de un lenguaje unívoco, por la realización física de un código lógico-formal.

Así las cosas, no podemos explicar lo digital simplemente como realidad física, aunque necesitemos entender su realización física. Precisamente, entendiendo la realización física de lo digital, podemos entender por qué lo digital es más que físico. Lo que percibimos físicamente como soporte de lo digital no nos explica por qué es una realidad que responde a un ordenamiento lógico-formal con realización física. Estamos ante la expresión de una realidad no física, o en sentido estricto, metafísica, inmersa en una realidad física. Los ceros y los unos mantienen unas relaciones según las reglas establecidas por el código, esto es netamente metafísico, pero las ocurrencias electrónicas son su manifestación física. Estas reglas y relaciones son igualmente formales. Por ello, entendemos que lo digital implica que las reglas y relaciones entre ceros y unos tienen una realización física concreta, y aunque no confundimos lo físico con lo digital, podemos entender la realización física de lo digital como efectos sobre el mundo físico. De aquí surge un nuevo aspecto: el problema del efecto.

Los efectos de dichas reglas son lo único que puede percibirse del universo digital en el mundo físico: como mínimo vemos y oímos sus efectos. Pero ni los ceros, ni los unos, y muchos menos sus combinaciones, nos son perceptibles directamente, solo son pensables. Del mismo modo, solo ciertos flujos electrónicos, principalmente lo que se presenta en la pantalla como luz para ser vista, son perceptibles por el hombre que, en este caso, se denomina por convención "usuario".

Cuando decimos que el universo digital está en nuestro mundo, apuntamos al hecho de que en el marco de esas reglas entre ceros y unos, puede darse un sinnúmero de situaciones que no se dan de ordinario en nuestro mundo salvo como efecto de este universo digital. Con ello no decimos que sea absolutamente ilimitado, porque lo digital no sale de los límites marcados por su realización física, como tampoco de los límites lógico-formales fijados por el sistema de reglas que lo determina. Podemos concluir, así, con tres condiciones de lo digital: la primera es la electricidad; la segunda son los dispositivos que la realizan y la tercera es la regulación de ceros y unos. Este es el universo digital en su simpleza y potencia.

\section{Los usos de lo digital}

Sin embargo, comprender el funcionamiento de lo digital no lleva directamente a comprender sus usos y las maneras en que la cotidianidad de lo humano puede transformarse. Este segundo componente del problema abrirá paso al juicio ético.

Debemos detallar el elemento del universo digital que es "enviado" desde nuestro mundo: el código. En efecto, cuando decíamos que los ceros y los unos 
se relacionaban según ciertas reglas, pero que ninguno de ellos tenía realidad física sino eléctrica, estábamos suponiendo este elemento aunque no plenamente. El código es un sistema singular de relaciones que hace que un dispositivo funcione de tal o cual manera. La forma más sencilla de entenderlo es como software, esto es, como programa. Cada programa, cada código, actualiza con precisión las relaciones entre ceros y unos; se trata de una manera-determinada por reglas- de relacionarse.

En un sentido amplio, los ceros y los unos solo existen como códigos singulares, como relaciones ¿Por qué? Porque, de hecho, si no se siguen las reglas del código en la ejecución, la máquina sencillamente se traba. Sin embargo, creemos que es importante hacer esta diferenciación dado que el código establece, en tanto que singularidad actual, el puente entre lo físico de lo digital y el mundo de la vida de los hombres ${ }^{1}$. Código es el firmware de un aparato, como el programa que convierte libras en pies, así como el sistema operativo del ordenador y el diferenciador de billetes de una máquina para pagar el parqueadero. Gracias al código, los aparatos electrónicos funcionan a nuestra conveniencia y puede decirse que entran al mundo humano.

Puesto que hemos pasado, en nuestro análisis, al lado del mundo humano, tenemos que comprender en él los efectos de lo digital. Para ello, recurrimos a dos sujetos que son quienes propiamente aprovechan y entran en comunicación con el universo digital desde el mundo humano. En cierto sentido, ellos son los creadores del universo digital, pero también sus beneficiarios (o "creaturas"): el usuario y el programador. Sin ellos resulta difícil entender lo digital en el mundo humano. El usuario es quien ajusta su cotidianidad en tanto sea usuario de múltiples dispositivos a través de variados programas; el programador, por su parte, es quien abre las posibilidades para el usuario. Las dos subjetividades son inseparables, al punto que resulta que una es condición de la otra. Efectivamente, hay usuarios que no son programadores, como lo somos la mayoría, pero todo programador es, necesariamente, un usuario. Esto hace del usuario la subjetividad básica de lo digital.

La característica definitoria de lo que significa ser un usuario digital es la de no tener acceso al código del dispositivo cuando está en funcionamiento; en un sentido amplio, todo lo digital se esconde para el usuario. Esto lo decimos, claro está, desde el lado del mundo humano, no desde el lado del universo digital. Solo los efectos de lo digital en el mundo se hacen patentes al usuario. Aun para el programador, porque no interactúa directamente con el código del lenguaje en el cual programa, puede entenderse que siempre hay un código que le queda oculto. Realmente, la misma idea de "interacción con un código" implica que no se está trabajando el programa como listado, sino que se está interactuando con el programa en ejecución, razón por la cual está necesariamente oculto como código: el usuario no se las ve con el código sino con sus efectos.

1 Este aspecto derivará en el problema de los niveles de codificación y la relación entre ellos, asunto que excede las posibilidades de esta disertación. 
Como ha de notarse, estamos retomando la última idea de la sección anterior, según la cual los ceros y los unos regulados no son perceptibles, solo pensados. Esto nos indica algo muy importante de la relación entre el universo digital y nuestro mundo: el código es un puente entre lo que el usuario espera percibir como efecto - por ejemplo, en la pantalla- y lo que el dispositivo electrónico tiene que realizar para que dicho efecto ocurra.

La realización física de lo digital es del interés de muy pocos. Lo que empieza a interesar es lo que puede hacerse con un aparato, en virtud de lo que el aparato mismo puede hacer: comunicarnos con otros a distancia, leer un libro, hacer un cálculo complejo, simular una realidad y similares. Para el aparato nada de eso tiene sentido ni significado, solo para el hombre que lo utiliza ${ }^{2}$. Un celular es, digitalmente, exactamente igual que un cajero automático, que un computador, que un reproductor de discos blu-ray; las diferencias de uso están del lado de lo humano, no del lado de lo digital. En el universo digital todo es exactamente igual: ceros y unos que se relacionan según ciertas reglas en una realización eléctrica singular. Pero en el mundo humano se abren posibilidades de acción, nuevas, renovadas y hasta inverosímiles o imposibles, por lo menos de un tiempo para atrás.

Tenemos así dos lados reconciliables por el código: un lado de flujos electrónicos que puede reducirse a ceros, unos y relaciones reguladas que se actualizan eléctricamente, y otro del mundo de la vida, la cotidianidad del usuario y del programador.

\section{La cotidianidad del usuario}

El tercer componente del problema es la relación entre el universo digital en sí y su respectivo usuario. Consideramos que si establecemos una clasificación de usuarios, podemos entender los grados de afección de lo digital en la cotidianidad de lo humano. Los dos primeros niveles son estándares, difícilmente imaginamos que alguien en el siglo XXI no pudiera rápidamente ver el mundo de ese modo. Pero los siguientes tres niveles sí marcan una diferencia grande. Los niveles que hemos identificado en la investigación son los siguientes:

- Click and play: Este es el nivel más básico que podemos encontrar. Se trata del hecho de oprimir un botón y disfrutar del aparato: encender la luz, la TV, el computador, etc. Pero no solo en referencia al inicio del aparato, sino también a los botones que lo controlan: cambiar el canal, subir el volumen, iniciar fax, marcar el teléfono, enviar un email. Básicamente, todo el internet depende de esta habilidad. Ahora bien, no podemos decir que por el hecho de poder encender la luz, ya la persona va a ser capaz de navegar por internet o enviar correos electrónicos o guardar números de teléfono en su móvil. Pero se trata de

2 Cf. Este punto de vista se basa en desarrollos a partir del experimento mental de Searle (1984) conocido como "El cuarto chino". 
un nivel en el cual la persona es, claramente, un usuario que utiliza los botones del aparato, tal y como estos fueron "concebidos", para lograr ciertos efectos. Y debe estar ya instalado y a disposición al usuario. Esto explica por qué una persona de ochenta años que siempre supo cómo contestar y marcar el teléfono pueda hoy, sin mayor sorpresa, enviar correos electrónicos. Se trata de un nivel no de conocimiento, sino de la manera de concebir el mundo: "yo oprimo tal o cual botón y tal o cual cosa tiene que pasar". Este nivel de botones, de teclas, de pulsaciones nos rodea por todas partes.

- Plug and play: Diferente es quien es capaz de instalar un artefacto; el DVD al televisor, por ejemplo. Aquí solo diferenciamos la necesidad de interactuar físicamente con el aparato. La impresora no se enchufa sola ni se alimenta de papel a sí misma. Las USB han facilitado mucho la vida, pero eso era lo que básicamente sucedía cuando enchufábamos al tomacorriente cualquier aparato eléctrico. Se conecta, se oprime un botón y listo. Pensamos que este nivel es superior porque, si bien implica una acción corporal y no digital, implica a su vez un paso atrás con respecto al anterior, en la medida en que es condición del click and play. Incluso, ciertas manipulaciones pueden cualificar mucho ese primer nivel, para lograr efectos más complejos o más detallados. El usuario no depende, así, de un técnico instalador.

- Follow instructions and play: Aquí estamos hablando, propiamente, del uso del software y de lo que consideramos propiamente un usuario digital. A este nivel, una persona es capaz, por ejemplo, de hacer uso de las diferentes herramientas de un procesador de palabras. No solo puede subrayar, resaltar o corregir la ortografía, sino que además puede generar fácilmente tablas de contenidos, índices, tipos de letras y demás. Lo importante es que el código, el software, pueda lograr ciertos efectos si el usuario sigue un sistema de reglas para ello, esto es, sus instrucciones de uso. Este sistema de reglas de programa está garantizado por el mismo sistema de reglas de programación, cuestión que no podemos perder de vista.

- Run and play: Aunque este nivel se parece al anterior, lo diferenciamos porque se trata de saber combinar resultados entre programas, lo que puede implicar la conversión de archivos o formatos de uno a otros programas. Esto supone que el usuario sea capaz de tomar los resultados que produce cada software y manipularlos para ser utilizados por otro software con otros fines. Aquí estamos al nivel de copiado de CD de música, su conversión y envío a otros aparatos, cambios de formato, incluso devueltos a una versión análoga diferente. La dificultad para diferenciar este nivel radica en que muchos procedimientos que hace unos años se hacían de este modo ya se encuentran en el nivel anterior, el tercero, follow instructions and play, porque se han hecho programas que abarcan interacción entre programas ${ }^{3}$. Yo diría que la cocina es toda a este nivel. Y en el futuro, muchas actividades de este nivel se encontrarán en programas que

3 Si se tiene en cuenta el desarrollo de la programación por objetos, se entiende que esto termine sucediendo con los programas. Estos empiezan a funcionar como piezas de construcción que se pueden combinar de muchas maneras a punto de que evite que el usuario tenga que comprender su funcionamiento. 
ya hacen todo por nosotros, o con los que es poco lo que debemos hacer. Si se toma conciencia de este nivel, vemos cómo puede suceder que el usuario se acerque o aleje del código. En efecto, llegar a este nivel implica entender algo del código para poder hacer este tipo de transferencias.

- Program and play: Aquí ya no se trata de usar programas sino de crear los programas para ser utilizados. El usuario deviene programador. El programador es, en este sentido, un usuario avanzado. La línea es confusa porque hoy en día los programadores utilizan piezas de programas ya hechas para armar programas más complejos. Linux Trovalds, por ejemplo, creador de Linux, no participa sino en el $2 \%$ de lo que hoy sería el Linux, porque otros programadores han participado en grupos de programación. Así como ya ningún ser humano podría hacer un televisor plasma desde cero, un programador no podría desarrollar desde cero un procesador de palabras, por ejemplo. De hecho, su actividad dependería de un lenguaje de programación, que seguramente él no hizo, o de un sistema operativo que soporta el lenguaje de programación que él utiliza. Al igual que los niveles anteriores, hay muchísimos más elementos de las etapas anteriores que se siguen usando, y seguramente con más complejidad. De hecho, en eso consiste gran parte de la programación actual, en saber reutilizar lo que otros han logrado y perfeccionarlo, haciéndolo más rico, más detallado o más preciso.

- Hack and play: Aquí es donde nos encontramos con un pensamiento fresco y un procedimiento nuevo. No es solo estudiar lo que otros han hecho sino la posibilidad de reconstruir lo que sea necesario para lograr un objetivo. Aquí es donde se ubican los desarrolladores de sistemas operativos, de lenguajes de programación, los programadores de nuevos equipos. Aquí, una vez más, empezamos a salir del "puro" del software y nos volvemos a encontrar con las máquinas, los metales, la electricidad y el código directamente. Y es que si se cambia la arquitectura de un ordenador, entonces necesitamos un nuevo sistema operativo, un nuevo driver, una nueva interfaz que nos permita comunicarnos con la máquina.

De los anteriores niveles podemos sacar ya algunas conclusiones importantes. Queremos resaltar brevemente algunas:

a. Ningún nivel es puro. Es decir, ningún usuario se mueve exclusivamente en un nivel.

b. Nunca hay un uso puro de códigos, de software, siempre necesitamos una mediación analógica: la mano, la vista, etc.

c. Cuando el usuario accede a un mayor nivel en el acceso al software, implica más acceso directo con la máquina, mayor cercanía al código.

d. El sistema de reglas de utilización del software no es el mismo que el sistema de reglas que hace posible el programa. Se relacionan, pero hay una diferencia importante entre las reglas para el usuario y las reglas de programación. 
e. Ninguno de los niveles implica, en sí, una moralidad. No encontramos que haya acciones a prohibir o a impedir.

Sin embargo, encontramos que hay prohibiciones, ilegalismos, etc. Lo que queremos hacer notar, para poder pasar a la siguiente sección, es que ninguna de las prohibiciones o infracciones pertenecen propiamente a la relación usuario digital-máquina. Todas ellas pertenecen al mundo humano, no al universo digital. Se trata de una imposición de regulaciones de un mundo que ya posee unas reglas propias y de diferente naturaleza de las de un flamante universo digital.

\section{Lo ético, lo digital y lo humano}

Solo un cuarto componente del problema nos permitirá diferenciar dónde ubicar el perjuicio y la utilidad de lo digital para la vida.

Queremos, para terminar, recoger estos prolegómenos en la siguiente idea: la eticidad de lo digital, la modificación que opera en la cotidianidad de los humanos, está directamente relacionada con el grado de acceso, esto es, de comprensión y manipulación del código. Dicho en otras palabras: la discusión ética de lo digital solo alcanzará su mayoría de edad cuando los implicados en ella sean plenos ciudadanos de un mundo, cuando manejen mínimamente el código. Con ello no queremos excluir otras variables o factores que determinen la eticidad de lo digital, simplemente queremos sentar un punto de partida consistente para avanzar en la discusión.

El usuario digital, entendemos mejor, no es una unidad sino que es una multiplicidad. Por lo menos, hemos identificado seis niveles de usuario y hemos propuesto algunas consecuencias. El ocultamiento del código es, propiamente, donde se establece el perjuicio o el beneficio para la vida. Hemos visto que, en principio, el usuario no puede tener acceso directo al código, pero también que el devenir programador del usuario hace que este tenga que vérselas con el código no ya como un agente pasivo o consumidor, sino como un agente activo. No será entonces un usuario ingenuo, menor de edad, que como un niño, tendría que tener normas dictadas por una especie de consciencia superior, sino que sería un usuario mayor de edad que puede regularse críticamente en una nueva cotidianidad digital

Todos recordarán de la película Matrix la diferencia entre Neo y los demás (Morpheus, Trinity, etc.). Hubo un momento en el que Neo tuvo acceso directo al código, todos los demás estaban completamente mediados. Todos padecían la simulación de la realidad física dentro de la Matrix, aunque hacían cosas que podían trasgredir un poco los límites de la realidad; estaban mediados por los efectos del código y asumían dichos efectos como lo único delante de ellos. Incluso, quien observaba tras la pantalla fuera de la Matrix para que pudieran entrar y salir no tenía pleno acceso al código. Solo Neo pudo hacer lo imposible, 
precisamente porque tenía acceso directo al código: Neo era el primer programador puro que accedía al código justo en su ejecución, era el ideal de usuario que ya no es usuario.

Este es el punto que deseamos acentuar: la lógica de lo digital es, como hemos descrito, una sencilla relación regulada de ceros y unos que se actualizan eléctricamente en un dispositivo particular. La cuestión es que ni la regulación ni los aparatos se hacen solos, cuestión obvia, aunque hasta ahora lo resaltemos. Esto trae muchos problemas por escudriñar, pero lo cierto es que tienen una peculiaridad que acontece para el mundo humano aunque no para el universo digital, y que solo podíamos ver hasta ahora:

Los objetos digitales son entes autónomos, son realidades autosuficientes que se independizan de su creador; de un modo relativo, claro está. Nadie duda que los ordenadores deban enchufarse y que las plantas de energía eléctrica se pongan en funcionamiento por la mano humana, pero una vez en ejecución (los programas corren), no se detienen: en muchos casos pueden esperar a que el usuario pulse alguna tecla, haga un movimiento; en otros, siempre están adelantando una función, una labor para los humanos. No tenemos que repetir incesantemente toda la génesis de los ordenadores cada vez que adquirimos uno nuevo, todo dispositivo nuevo llega completamente listo para ser utilizado y el usuario no necesita más que oprimir un botón: nada de programación, solo plug and play, en el mejor de los casos.

Tal vez la consecuencia de esta verdad de la autonomía del software es lo que nos retiene de hacer un recorrido sobre lo que tenemos todo el tiempo delante de nosotros. ¿Por qué? Porque el programa, el software, el código, se presenta como el genio dentro de la botella, como aquello que va a satisfacer todos nuestros deseos. Por sí solo puede imprimir las cosas bien, sin que tengamos que repetir las hojas de la máquina de escribir hasta que salgan con el mínimo de errores o sin ellos. El programa, al funcionar por sí mismo, hace las tareas por nosotros, cada vez intervenimos menos.

Dicho así suena muy loable, deseable y suscitará la pregunta: “quién quiere cuestionar o poner en ridículo un logro tan importante de la cultura y de la humanidad?", pero el problema es que en la medida en que lo digital nos facilita la vida, en que satisface más nuestros deseos sin que tengamos que intervenir-o intervengamos muy poco-, en esa misma medida o incluso en una proporción aún mayor, nos alejamos del código, de su complejidad y de lo que significa lograr esos efectos. Este es, creemos, el verdadero perjuicio de lo digital: la enajenación con respecto al código. ¿Por qué?

Porque no superar ni llegar al nivel run and play, que describíamos antes como la capacidad de combinar efectos de programas a través de ciertos procesos de transformación, significa mantenernos alejados de lo que está detrás de la pantalla. Con esta idea no queremos empezar una especie de paranoia en la que supongamos que hay un nuevo fantasma dentro de la máquina. Pero esta 
preocupación sí es paralela a la de Richard Stallman con respecto al software libre: si no sabemos lo que hay en el código que usamos, no somos verdaderamente libres. Si preferimos aceptar el distanciamiento con respecto al código, entonces estamos aceptando no ser libres porque ya no sabemos qué están haciendo en "nuestros" equipos; podemos ver lo que sucede en la pantalla pero no saber lo que sucede detrás de ella.

Sin embargo, ¿no es propia de nuestras sociedades la especialización del trabajo? Es decir, ¿no es crucial para una sociedad de libre mercado el hecho de que yo no tenga que preocuparme por saber cómo construir una casa para tener una, o saber cómo funciona un carro para manejarlo? La especialidad en el pensamiento, en la acción y en el trabajo es perfectamente entendible, pero otra cosa es cuando vemos que lo digital aparece por todas partes.

Nos parece que si bien el código, en tanto que formal, no cumple el papel del lenguaje humano en lo que se refiere a las variaciones y vibraciones entre significantes y significados, al punto de que el sentido en el lenguaje es completamente libre, el código, a través de sus efectos, sí está permeando todos los espacios de la cotidianidad, del mismo modo en que el lenguaje lo hace. Pero debemos especificar más: el código sería un lenguaje unívoco, artificial y se actualiza en las máquinas, si es que cabe hablar de lenguaje cuando nos referimos al código. Pero el lenguaje humano es equívoco, sin autor o dueño y se actualiza en los hablantes. El lenguaje es correlativo al mundo de la vida, a la cotidianidad, y el código es un elemento, de hecho, es un resultado del mundo de la vida. Así su eticidad tendrá plena vigencia cuando no sea un misterio.

\section{Referencias}

Adorno, Theodor (1967). "La industria cultural". En: Edgar Morin y Theodor Adorno, La industria cultural, Buenos Aires: Galerna.

Spinoza, B. (2000). Ética demostrada según el orden geométrico (Atilando Domíguez, trad.). Madrid: Editorial Trotta.

Heidegger, M. (2007). Filosofía, Ciencias y Técnica (Francisco Soler, trad.). Santiago de Chile: Editorial Universitaria.

Ihde, D. (1990). Technology and the lifeworld: From garden to earth. Bloomington: Indiana University Press.

Searle, J. (1984). Minds, brains and science. Cambridge: Harvard University Press. . (2010). Making the social world: The structure of human civilization. Nueva York: Oxford University Press. 